\title{
Boolean Conservative Extension Results for some Modal Relevant Logics
}

\author{
Edwin D. Mares* \\ Philosophy Programme \\ and Centre for Logic, Language, and Computation \\ Victoria UNiversity of Wellington \\ Edwin.Mares@vuw.ac.nz \\ Koji Tanaka \\ Philosophy Department \\ University of Auckland \\ k.tanaka@auckland.ac.nz \\ Received by Greg Restall \\ Published September 22, 2010 \\ http://www.philosophy.unimelb.edu.au/aj1/2010 \\ (C) 2010 Edwin D. Mares and Koji Tanaka
}

Abstract: This paper shows that a collection of modal relevant logics are conservatively extended by the addition of Boolean negation.

\section{i Dedication}

This paper is dedicated to the memory of Bob Meyer. Bob was our friend and colleague. We miss him greatly.

\section{INTRODUCTION}

This paper is a sequel to [12]. That paper is concerned with modal relevant logic, in particular logics close to Meyer's system NR. NR was first developed as a tool to analyze the logic of entailment. The notion of entailment had been formalized by Anderson and Belnap in logic the logic E (for 'entailment') that had a conditional that incorporates both the notion of a relevant connection between antecedent and consequent and the concept of a necessary connection

* Our greatest debt, of course, is to Bob Meyer who formulated the central question of the paper and discussed it with Mares a great deal back in the early I990s. In addition, we thank Dov Gabbay, Hans-Jürgen Ohlbach, Rob Goldblatt, Jill LeBlanc, Greg Restall, John Slaney, and Richard Sylvan for discussions related to this problem. 
between the two. It is, in short, the relevant version of strict implication. This dual nature of entailment, however, suggests that the two notions can be separated, using a contingent relevant implication and a necessity operator. Taking up this suggestion, Meyer created NR, which combined the logic R (the logic of contingent relevant implication) with the modal logic $\mathrm{S}_{4}$. But it turned out that the fragment of this logic that is supposed to mimic E-conjunction, disjunction, negation, and necessary relevant implication—is slightly stronger than E.

NR turned out, however, to be interesting in its own right. Its approach to combining relevance and modality has been adopted and generalized by several authors (see, e.g., [2] and [4]). It is the ancestor of today's relevant temporal logics [20], relevant deontic logics [5], and relevant counterfactual logics (see [2]), and so on. One such related logic is $\mathrm{R}_{4}$. $\mathrm{R}_{4}$ differs from NR in that it contains all instances of the following scheme:

$$
(!) \square(\mathrm{A} \vee \mathrm{B}) \rightarrow(\diamond \mathrm{A} \vee \square \mathrm{B})
$$

The original interest in adding this scheme is the $\square, \wedge, \vee, \neg$ fragment of the resulting logic is just $S_{4}$. Thus, from the point of view of $R_{4}$, we might think of $\mathrm{S}_{4}$ as a good logic-it is only deficient in that it does not have a real implication connective.

In the previous paper, we did not discover whether $\mathrm{CR}_{4}-\mathrm{R}_{4}$ together with a boolean negation-is a conservative extension of $\mathrm{R}_{4}$. And this paper will not provide an answer to this question either. But it will look at several other systems very close to $\mathrm{R}_{4}$, that include the scheme (!), and prove that the boolean extension of each of these is conservative.

The original motivation for relevant logic is to avoid the paradoxes of implication, some of which essentially involve negation, such as ex falso $((p \wedge \neg p) \rightarrow$ q). To avoid these paradoxes, relevant logic replaces boolean negation with a weaker DeMorgan negation. So, the question arises, whether the addition of a boolean negation will affect the rest of a relevant logic-will it alter the properties and relationships between implication, DeMorgan negation, and the other connectives? In [I3] and [I4] Meyer and Routley discovered that boolean negation can be conservatively added to $\mathrm{R}$. This did not turn out to be the case, however, for NR [12] or E [9]. So, it seems that at least some modal relevant logics are fragile when it comes to the addition of a boolean negation. The formula that was used to prove that CNR and CE are not conservative extensions of their non-boolean kin is

$\neg \square(A \vee B) \vee(\diamond A \vee \square B)$

This formula is a theorem of CNR and CE but not of NR or E. It is, however, a theorem of all of the systems that we examine. This is one reason why the question that we are solving is interesting. 
Another motivation for this paper comes from proof theory. In [3] and [ [5], J.M. Dunn and Grigori Mints present sequent calculi for $\mathrm{R}$ that have two sorts of structural connectives-extensional and intensional connectives. Nuel Belnap has adopted and generalized this idea in his display logic. Belnap uses not only structural connectives that correspond to conjunction and disjunction, but he also employs structural negations. The advantage of having a boolean (or extensional) structural negation is that it can be used together with extensional conjunction and disjunction in versions of the antilogism rule, such as

$$
\frac{\mathrm{A} \circ_{e} \mathrm{~B} \vdash \mathrm{C}}{\mathrm{A} \vdash *_{e} \mathrm{~B} \circ_{e} \mathrm{C}} \text { and } \frac{\mathrm{A} \vdash \mathrm{B} \circ_{e} \mathrm{C}}{\mathrm{A} \circ_{e} *_{e} \mathrm{~B} \vdash \mathrm{C}}
$$

where $\circ_{e}$ is the extensional conjunction or disjunction (depending on whether it is on the left or right side of the turnstile) and $*_{e}$ is the extensional structural negation. Belnap has used the Routley-Meyer proof of conservative extension to prove that this use of extensional negation is harmless. To do this, he uses the fact that proofs in his display logic can be translated straightforwardly into valid proofs in the Hilbert system for CR (see [I] \$62).

\section{The Logics}

The logic that we use as our base logic is called R.K. ${ }^{-}$. The minus sign indicates that the following thesis of $\mathrm{R}_{4}$ is missing from $\mathrm{R} . \mathrm{K}^{-}$(and from all the other logics that we examine here):

$$
\square(\mathrm{A} \rightarrow \mathrm{B}) \rightarrow(\square \mathrm{A} \rightarrow \square \mathrm{B}) .
$$

The reason that we do not include it is that its associated semantic postulate ${ }^{2}$ cannot be proven to hold in the canonical model of section 6 below. Whether the logics that include this thesis (as well as (!) and the other axioms of our base system) are conservatively extended by the addition of boolean negation is still and open question.

Although the loss of $\square(A \rightarrow B) \rightarrow(\square A \rightarrow \square B)$ clearly weakens the logic, it does not do so very much. All the logics that we examine are closed under the rule $\mathrm{RM}$, that is, $\vdash \mathrm{A} \rightarrow \mathrm{B} \Longrightarrow \vdash \square \mathrm{A} \rightarrow \square \mathrm{B}$ and contain the thesis $(\square \mathrm{A} \wedge \square \mathrm{B}) \rightarrow \square(\mathrm{A} \wedge \mathrm{B})$. Together with some standard R-moves we can prove $(\square(\mathrm{A} \rightarrow \mathrm{B}) \wedge \square \mathrm{A}) \rightarrow \square \mathrm{B}$.

The language $\mathcal{L}$ contains a non-empty set of propositional variables, the unary connective $\neg$, the binary connectives $\wedge$, $\circ$ (fusion or intensional conjunction), and $\rightarrow$, and parentheses. Standard formation rules hold. We also define the following connectives:

$$
\mathrm{A} \vee \mathrm{B}={ }_{\mathrm{df}} \neg(\neg \mathrm{A} \wedge \neg \mathrm{B})
$$

\footnotetext{
${ }^{\mathrm{I}}$ In the case of $\mathrm{E}$, whose boolean extension is not conservative, Belnap uses another method. The use of conservative extension results is not essential, but very convenient.

${ }^{2}$ Which is $\exists x(\operatorname{Rabx} \wedge S x c) \Rightarrow \exists x \exists y(S a x \wedge$ Sby $\wedge$ Rxyc $)$, and is known, for obvious reasons, as "Hume's dictum".
} 


$$
\begin{gathered}
\mathrm{A} \leftrightarrow \mathrm{B}=_{\mathrm{df}}((\mathrm{A} \rightarrow \mathrm{B}) \wedge(\mathrm{B} \rightarrow \mathrm{A})) \\
\diamond \mathrm{A}=_{\mathrm{df}} \neg \square \neg \mathrm{A} \\
\mathrm{A} \circ \mathrm{B}=_{\mathrm{df}} \neg(\mathrm{A} \rightarrow \neg \mathrm{B})
\end{gathered}
$$

The following is an axiomatization of R.K ${ }^{-}$:

\section{Axiom Schemes}

I. $A \rightarrow A$

2. $(A \rightarrow(B \rightarrow C)) \rightarrow(B \rightarrow(A \rightarrow C))$

3. $(\mathrm{A} \rightarrow \mathrm{B}) \rightarrow((\mathrm{B} \rightarrow \mathrm{C}) \rightarrow(\mathrm{A} \rightarrow \mathrm{C}))$

4. $(\mathrm{A} \rightarrow(\mathrm{A} \rightarrow \mathrm{B})) \rightarrow(\mathrm{A} \rightarrow \mathrm{B})$

5. $A \rightarrow(A \vee B) ; B \rightarrow(A \vee B)$

6. $(\mathrm{A} \wedge \mathrm{B}) \rightarrow \mathrm{A} ;(\mathrm{A} \wedge \mathrm{B}) \rightarrow \mathrm{B}$

7. $((\mathrm{A} \rightarrow \mathrm{C}) \wedge(\mathrm{B} \rightarrow \mathrm{C})) \rightarrow((\mathrm{A} \vee \mathrm{B}) \rightarrow \mathrm{C})$

8. $((\mathrm{A} \rightarrow \mathrm{B}) \wedge(\mathrm{B} \rightarrow \mathrm{C})) \rightarrow(\mathrm{A} \rightarrow(\mathrm{B} \wedge \mathrm{C}))$

9. $(A \wedge(B \vee C)) \rightarrow((A \wedge B) \vee(A \wedge C))$

Iо. $\neg \neg \mathrm{A} \leftrightarrow \mathrm{A}$

II. $(\mathrm{A} \rightarrow \neg \mathrm{B}) \rightarrow(\mathrm{B} \rightarrow \neg \mathrm{A})$

I2. $(\square \mathrm{A} \wedge \square \mathrm{B}) \rightarrow \square(\mathrm{A} \wedge \mathrm{B})$

I3. $\square(A \vee B) \rightarrow(\diamond A \vee \square B)$

\section{Rules}

$$
\begin{aligned}
& \frac{\vdash A \rightarrow B}{} \frac{\vdash A}{\therefore}(\mathrm{MP}) \\
& \quad \vdash A \\
& \frac{\vdash B}{\therefore \vdash A \wedge B}(\mathrm{Adj}) \\
& \frac{\vdash A \rightarrow B}{\vdash \square A \rightarrow \square B}(\mathrm{RM})
\end{aligned}
$$




$$
\frac{\vdash \mathrm{A}}{\therefore \vdash \mathrm{A}}(\mathrm{Nec})
$$

We can extend R.K $\mathrm{K}^{-}$by the addition of any subset of the following list of axiom schemes and the conservative extension theorem will hold of the resulting logic:

\begin{tabular}{cc}
\hline SCHEME & NAME \\
\hline$\square \mathrm{A} \rightarrow \diamond \mathrm{A}$ & $\mathrm{D}$ \\
$\square \mathrm{A} \rightarrow \mathrm{A}$ & $\mathrm{T}$ \\
$\square \mathrm{A} \rightarrow \square \square \mathrm{A}$ & 4 \\
$\mathrm{~A} \rightarrow \square \triangleright \mathrm{A}$ & $\mathrm{B}$ \\
\hline
\end{tabular}

In what follows we make use of the following lemma.

LEMMA I The following are provable in R.K $K^{-}$:

(i) $((\mathrm{B} \rightarrow \mathrm{C}) \vee(\mathrm{D} \rightarrow \mathrm{E})) \rightarrow((\mathrm{B} \wedge \mathrm{D}) \rightarrow(\mathrm{C} \vee \mathrm{E}))$;

(ii) $((\mathrm{A} \rightarrow \mathrm{B}) \wedge \mathrm{A}) \rightarrow \mathrm{B}$;

(iii) $(\mathrm{A} \rightarrow \mathrm{B}) \rightarrow(\mathrm{A} \rightarrow(\mathrm{A} \circ \mathrm{B}))$;

(iv) $(\mathrm{A} \rightarrow(\mathrm{B} \rightarrow \mathrm{C})) \rightarrow((\mathrm{A} \rightarrow \mathrm{B}) \rightarrow(\mathrm{A} \rightarrow \mathrm{C}))$;

(v) $\mathrm{A} \rightarrow(\mathrm{B} \rightarrow(\mathrm{A} \circ \mathrm{B}))$;

(vi) $(\mathrm{A} \rightarrow(\mathrm{B} \rightarrow \mathrm{C})) \rightarrow((\mathrm{A} \circ \mathrm{B}) \rightarrow \mathrm{C})$;

(vii) $(\mathrm{A} \rightarrow \neg \mathrm{A}) \rightarrow \neg \mathrm{A}$;

(viii) $A \vee \neg A$.

Proof: Straightforward.

\section{Normal Theories}

In this section we prove that $\mathrm{R}^{-} \mathrm{K}^{-}$and the other logics are characterized by the class of their prime consistent theories that contain all their theorems. The proof is by means of Meyer's method of metavaluations. A metavaluation is a process whereby we take a prime theory containing all the theorems of a logic and shrink it to a consistent such theory. But before we can explain properly what a metavaluation is, we to give some definitions and to prove a few lemmas.

Where $\Gamma$ is a set of formulas, $\Sigma \vdash_{\Gamma} \Delta$ iff $\exists A_{1} \ldots A_{n} \in \Sigma \exists B_{1} \ldots B_{m} \in$ $\Delta\left(\left(A_{1} \wedge \cdots \wedge A_{n}\right) \rightarrow\left(B_{1} \vee \cdots \vee B_{m}\right) \in \Gamma\right)$. A set of formulas $\Sigma$ is said to be a $\Gamma$-theory if, for any formula $A$, if $\Sigma \vdash_{\Gamma} A$, then $A \in \Sigma$. $\Sigma$ is said to be prime 
if for any disjunction $A \vee B \in \Sigma$, at least one of $A$ or $B$ is in $\Sigma$. And a pair of sets of formulas $(\Sigma, \Delta)$ is called independent if $\Sigma \nvdash_{\Gamma} \Delta$. A theory is regular if it contains all the theorems of R.K. $\mathrm{K}^{-}$and it is normal if it is prime, regular, and negation consistent. Where $\Gamma$ is just the set of R.K $\mathrm{K}^{-}$theorems, we call $\Gamma$-theories 'R.K $\mathrm{K}^{-}$-theories' or merely 'theories'. If $(\Sigma, \Delta)$ is R.K ${ }^{-}$-independent, then we merely say ' $(\Sigma, \Delta)$ is independent'. We only use the notion of independence, not the more general notion of $\Gamma$-independence in this section, but when it comes to proving completeness we will need the more general notion.

The following lemma is the standard Lindenbaum lemma for relevant logics, due originally to Dov Gabbay and Nuel Belnap.

LEMMA 2 If $\Gamma$ is a regular R.K $K^{-}$theory and $(\Sigma, \Delta)$ is $\Gamma$-independent, then there is a prime theory $\Sigma^{\prime}$ extending $\Sigma$ such that $\left(\Sigma^{\prime}, \Delta\right)$ is $\Gamma$-independent.

We now prove some preliminary lemmas.

LEMMA 3 If $\Gamma$ is a prime theory and there is a $\square \mathrm{A} \in \Gamma$ but no $\diamond \mathrm{B}$ is in $\Gamma$, then for all formulas $\mathrm{C}, \square \mathrm{C} \in \Gamma$.

Proof: Suppose that $\Gamma$ is a prime theory and there is a $\square A \in \Gamma$ but no $\diamond B$ is in $\Gamma$. By axiom 5, $A \rightarrow(A \vee C)$ and so by RM, $\square A \rightarrow \square(A \vee C)$. Moveover, by axiom $\mathrm{I}_{5}, \square(A \vee C) \rightarrow(\diamond A \vee \square C)$ and so $\square A \rightarrow(\diamond A \vee \square C)$. Since $\Gamma$ is a theory, $\diamond A \vee \square C \in \Gamma$. But, by hypothesis, $\diamond A \notin \Gamma$. Since $\Gamma$ is prime, $\square C \in \Gamma$.

As usual, we define $\square^{-1} \Gamma$ to be the set of all formulas $A$ such that $\square A \in \Gamma$ and $\diamond^{-1} \Gamma$ to be the set of all formulas $B$ such that $\diamond B \in \Gamma$.

Lemma 4 If $\Gamma$ is a prime theory, then $\square A \in \Gamma$ iff for all prime theories $\Gamma^{\prime}$ such that $\square^{-1} \Gamma \subseteq \Gamma^{\prime} \subseteq \diamond^{-1} \Gamma, A \in \Gamma^{\prime}$.

Proof: $\Rightarrow$ obvious.

$\Leftarrow$ Case I. Suppose that there are no formulas B such that $\diamond B \in \Gamma$. Then there are no theories that are a subset of $\diamond^{-1} \Gamma$. But by Lemma 3 for every formula $C \square C \in \Gamma$.

Case 2. There is at least one formula $B$ such that $\diamond B \in \Gamma$.Suppose that $\square A \notin$ $\Gamma$. Let $\Delta_{\Gamma}=(\{A\} \cup\{\mathrm{B}: \diamond \mathrm{B} \notin \Gamma\})$. We show that $\left(\square^{-1} \Gamma, \Delta_{\Gamma}\right)$ is independent. Suppose not. There there are some $G_{1}, \ldots, G_{n} \in \square^{-1} \Gamma$ and $B_{1}, \ldots, B_{m} \in\{B$ : $\diamond B \notin \Gamma\}$ such that $\left(G_{1} \wedge \cdots \wedge G_{n}\right) \rightarrow\left(A \vee B_{1} \vee \cdots \vee B_{m}\right)$ is a theorem of R.K $\mathrm{K}^{-}$. Then, by the rule RM, axioms 3, I3, I4, I5 (and a few applications of modus ponens), $\square\left(G_{1} \wedge \cdots \wedge G_{n}\right) \rightarrow\left(\square A \vee \diamond B_{1} \vee \cdots \vee \diamond B_{m}\right)$. Thus, since $\Gamma$ is a prime theory, either $\square A \in \Gamma$ or at least one of $\diamond B_{1}, \ldots, \diamond B_{m}$ is in $\Gamma$. But, by hypothesis, none of these formulas are in $\Gamma$ and so by reductio we conclude that $\left(\square^{-1} \Gamma, \Delta_{\Gamma}\right)$ is independent as required.

By Lemma 2, we can expand $\square^{-1} \Gamma$ to a prime theory $\Gamma^{\prime}$ such that $\left(\Gamma^{\prime}, \Delta_{\Gamma}\right)$ is independent, i.e. $\square^{-1} \Gamma \subseteq \Gamma^{\prime} \subseteq \diamond^{-1} \Gamma$ and $A \notin \Gamma^{\prime}$. 
Now we can give the definition of a metavaluation and proceed to the main part of the proof.

DEFINITION 5 (Kripke Metavaluation) For a regular prime theory $\Gamma$, we define $\models_{M}$ as follows: (Mp) for all propositional variables $p, \Gamma \models_{M} p$ iff $p \in \Gamma$; (MA) $\Gamma \models_{M} A \wedge B$ iff $\Gamma \models_{M} A$ and $\Gamma \models_{M}$ B; $(M \neg) \Gamma \models_{M} \neg A$ iff (i) $\neg A \in \Gamma$ and (ii) $\Gamma \nvdash_{M} A$; $(M \rightarrow) \Gamma \models_{M} A \rightarrow B$ iff (i) $A \rightarrow B \in \Gamma$ and (ii) either $\Gamma \nvdash_{M} A$ or $\Gamma \models_{M}$ B; $(M \square) \Gamma \models_{M} \square A$ iff $\forall \Theta\left(T_{M} \Gamma \Theta \Rightarrow \Theta \models_{M} A\right.$ ) (where $T_{M} \Gamma \Theta$ iff $\left.\square^{-1} \Gamma \subseteq \Theta \subseteq \diamond^{-1} \Gamma\right)$.

This notion of a metavaluation is named after Kripke because it in effect constructs a Kripke model out of the set of prime regular theories and reduces the whole set simultaneously to normal theories.

We now prove that for each regular prime theory $\Gamma, M(\Gamma)=\left\{A: \Gamma \models_{M} A\right\}$ is a normal theory. It is clear from $(M \wedge)$ that $M(\Gamma)$ is negation consistent, $(\mathrm{M} \wedge)$ indicates that it is closed under conjunction, and $(\mathrm{M} \rightarrow)$ entails that it is closed under modus ponens. But we still need to show that it is a prime, regular theory.

Following standard practice in relevant logic, we define $\Gamma^{*}=\{A: \neg A \notin \Gamma\}$. The following lemma can be proven in exactly the same was as it is in [8]:

LEMMA 6 Let $\Gamma$ be a prime regular theory, then $\Gamma^{*} \subseteq \mathrm{M}(\Gamma) \subseteq \Gamma$.

Lemma 6 proves that $M(\Gamma)$ is negation complete. For suppose that $A \notin$ $M(\Gamma)$. Then, by Lemma $6, A \notin \Gamma^{*}$, so by definition of $*, \neg A \in \Gamma$. Hence by our hypothesis and $(M \neg), \neg A \in M(\Gamma)$.

Lemma 6 also allows us to prove that $M(\Gamma)$ is prime. For suppose that $A \vee B \in \Gamma$ and suppose that $A \notin M(\Gamma)$ and $B \notin M(\Gamma)$. Then, $\neg A \in \Gamma$ and $\neg \mathrm{B} \in \Gamma$. So, by $(\mathrm{M} \neg), \neg \mathrm{A} \in \mathrm{M}(\Gamma)$ and $\neg \mathrm{B} \in \mathrm{M}(\Gamma)$. By $(\mathrm{M} \wedge) \neg \mathrm{A} \wedge \neg \mathrm{B} \in \Gamma$. But, by hypothesis and the definition of $\vee, \neg(\neg A \wedge \neg B) \in \Gamma$, contradicting the fact that $M(\Gamma)$ is negation consistent.

Axioms I-II can be proved to hold in $M(\Gamma)$ just as in $[8]$. We need to show that axioms $\mathrm{I} 2$ and $\mathrm{I} 3$ also hold in metavaluations.

LEMMA 7 If $A$ is an instance of axiom $I_{2}$ or $\mathrm{I}_{3}$, then $\Gamma \models_{M} A$, where $\Gamma$ is a regular prime theory.

Proof: (Axiom I2) Let $A$ be $(\square B \wedge \square C) \rightarrow \square(B \wedge C)$. Since $\Gamma$ is regular, $A \in \Gamma$. Now we suppose that $\Gamma \models_{M} \square \mathrm{B} \wedge \square \mathrm{C}$, that is $\Gamma \models_{M} \square \mathrm{B}$ and $\Gamma \models_{M} \square \mathrm{C}$. So, by $(\mathrm{M} \square)$, for all $\Gamma^{\prime}$ such that $\mathrm{T}_{M} \Gamma \Gamma^{\prime}, \Gamma^{\prime} \models_{M} \mathrm{~B}$ and $\Gamma^{\prime} \models_{M} \mathrm{C}$. Thus, for each such $\Gamma^{\prime}, \Gamma^{\prime} \models_{M} \mathrm{~B} \wedge \mathrm{C}$. So by $(\mathrm{M} \square) \Gamma \models_{M} \square(\mathrm{B} \wedge \mathrm{C})$. So $\Gamma \models_{M}(\square \mathrm{B} \wedge \square \mathrm{C}) \rightarrow \square(\mathrm{B} \wedge \mathrm{C})$.

(Axiom 13 ) Let $A$ be $\square(B \vee C) \rightarrow(\diamond B \vee \square C)$. Since $\Gamma$ is regular, $A \in \Gamma$. Now we suppose that $\Gamma^{\prime} \models_{M} \square(B \vee C)$. Thus, by (M $\square$ ), for all for all $\Gamma^{\prime}$ such that $T_{M} \Gamma \Gamma^{\prime}, \Gamma^{\prime} \models_{M} B \vee C$. Since $\Gamma^{\prime}$ is prime, either $\Gamma^{\prime} \models_{M} B$ or such that $\mathrm{T}_{M} \Gamma \Gamma^{\prime}, \Gamma^{\prime} \models_{M}$ C. If for at least one such $\Gamma^{\prime} \Gamma^{\prime} \models_{M} B$, then $\Gamma \models_{M} \diamond \mathrm{B}$ and hence $\Gamma \models_{M} \diamond \mathrm{B} \vee \square \mathrm{C}$. If, on the other hand, there is no $\Gamma^{\prime}$ such that $\Gamma^{\prime} \models_{M} B$, then all of them are such that $\Gamma^{\prime} \models_{M} C$ and so, by $(M \square), \Gamma \models_{M} \square C$ and hence $\Gamma \models_{M} \diamond \mathrm{B} \vee \square \mathrm{C}$. 
LEMMA 8 (i) If for all prime regular theories $\Gamma, A \rightarrow B \in M(\Gamma)$, then $\square A \rightarrow \square B \in$ $M(\Gamma)$; (ii) iffor all prime regular theories $\Gamma, A \in M(\Gamma)$, then $\square A \in \Gamma$.

Proof: (i) Suppose that or all prime regular theories $\Gamma, A \rightarrow B \in M(\Gamma)$. Then for all such $\Gamma, A \rightarrow B \in \Gamma$ and if $\Gamma \models_{M} A$, then $\Gamma \models_{M}$ B. Now, consider an arbitrary prime regular theory $\Theta$ such that $\Theta \models_{M} \square A$. Then for all $\Xi$ such that $T_{M} \Theta \Xi$, $\Xi \models_{M}$ A. Hence, $\Xi \models_{M}$ B for all such $\Xi$. Therefore, by Lemma $4, \Theta \models_{M} \square$ B, and so $\Theta \models_{M} \square \mathrm{A} \rightarrow \square \mathrm{B}$.

(ii) Follows directly from Lemma 4

THEOREM 9 R.K. $K^{-}$is characterized by the class of its normal theories, that is, $\vdash_{R . K^{-}}$A iff $A$ is in every normal theory of $R . K^{-}$.

\section{Simplified Semantics}

The semantics that we will use is a version of the simplified semantics. The simplified semantics for relevant logics started out as a semantics for the boolean extensions of relevant logics. Hence, it was originally called a "boolean semantics". We will use the name simplified semantics, due to [16], because we wish to show that the non-boolean logic R.K. $\mathrm{K}^{-}$is complete over it. We will then use this fact to prove that the boolean extension is conservative.

But, before we present the simplified semantics, we will present the standard (or "non-simplified") model theory. An R.K $\mathrm{K}^{-}$frame is a structure $\mathcal{F}=$ $\langle W, 0, R, S, *\rangle$ such that $W$ is a non-empty set (of "worlds"), 0 is a non-empty subset of $W, R \subseteq W^{3}, S \subseteq W^{2}$, and $*$ is a unary operator on $W$, such that the following definitions and semantic postulates hold:

$$
\begin{gathered}
a \leqslant b={ }_{d f} \exists x(x \in 0 \wedge R x a b) \\
T a b={ }_{d f} S a b \wedge S a^{*} b^{*}
\end{gathered}
$$

SPi Raaa

$\mathrm{SP}_{2} \mathrm{Rabc} \Rightarrow \mathrm{Rbac}$

$\mathrm{SP}_{3} \exists x(\operatorname{Rabx} \wedge \mathrm{Rxcd}) \Rightarrow \exists x(\operatorname{Rac} x \wedge \mathrm{Rxbd})$

$\mathrm{SP}_{4} \leqslant$ is a partial order

$\mathrm{SP}_{5}(\mathrm{a} \leqslant \mathrm{b} \wedge \mathrm{Rbcd}) \Rightarrow \operatorname{Racd}$

$\mathrm{SP} 6(\mathrm{a} \leqslant \mathrm{b} \wedge \mathrm{Sbc}) \Rightarrow \mathrm{Sac}$

$\mathrm{SP}_{7} \mathrm{Rabc} \Rightarrow \mathrm{Rac}^{*} \mathrm{~b}^{*}$

SP8 $a^{* *}=a$

SP9 $\mathrm{Sab} \Rightarrow \exists x(x \leqslant b \wedge \mathrm{Tax})$ 
SPIo $(a \in 0 \wedge T a b) \Rightarrow b \in 0$

An R.K. $\mathrm{K}^{-}$model is a pair $\langle\mathcal{F}, v\rangle$, where $\mathcal{F}$ is an $\mathrm{R} . \mathrm{K}^{-}$frame and $v$ is a function from propositional variables to sets of worlds closed upwards under $\leqslant . \quad v$ is extended to a satisfaction relation $\models_{v}$ (written merely as ' $\models$ ' when no confusion will result) according to the following inductive definition:

- $a \models p$ iff $a \in v(p)$

- $a \models A \wedge B$ iff $a \models A$ and $a \models B$

- $a \models \neg A$ iff $a^{*} \not \models A$

- $a \models A \rightarrow B$ iff $\forall x \forall y((\operatorname{Raxy} \wedge x \models A) \Rightarrow y \models B)$

- $a \models \square A$ iff $\forall x(\operatorname{Sax} \Rightarrow x \models A)$

A formula $A$ is valid on a model if for all $a \in 0, a \models A$.

A hereditariness theorem holds of all R.K $\mathrm{K}^{-}$models. That is, if $\mathrm{a} \models A$ and $a \leqslant b$, then $b \models A$, for all formulas $A$ and all worlds $a$ and $b$.

The following lemma is useful when proving soundness results:

LEMma io (Semantic Entailment) Let $\mathcal{M}=\langle W, 0, R, S, *, v\rangle$. Suppose that for all $\mathrm{a} \in \mathrm{W}$, if $\mathrm{a} \models \mathrm{A}$, then $\mathrm{a} \models \mathrm{B}$. Then, $\mathrm{A} \rightarrow \mathrm{B}$ is valid on $\mathcal{M}$.

A simplified R.K $\mathrm{K}^{-}$frame is an R.K $\mathrm{K}^{-}$frame that obeys the condition SPII:

$$
\text { (SPII) } a \leqslant b \Rightarrow a=b
$$

In other words, in simplified frames (and hence in simplified models) the partial order on worlds is an antichain.

In R.K $\mathrm{K}^{-}$models, the defined relation $\mathrm{T}$ acts like a modal accessibility relation in Kripke's sense. Whereas the derived truth condition for $\diamond$ is

$$
a \models \diamond A \text { iff } \exists x\left(\operatorname{Sa}^{*} x \wedge x^{*} \models A\right)
$$

we can prove that

$$
a \models \diamond A \text { iff } \exists x(\operatorname{Tax} \wedge x \models A)
$$

as well as

$$
a \models \square A \text { iff } \forall x(\operatorname{Tax} \Rightarrow x \models A) .
$$

We can, with a few modifications, eliminate $S$ in favour of $T$ and make our semantics much more Kripkean [7]. But in simplified models $S$ collapses into $\mathrm{T}$, and we get the Kripkean truth conditions automatically.

The following theorem was proven in [I2]. We repeat the proof here because of its importance for our completeness proof. 
THEOREM II (Clustering) If $\mathrm{a} \in \mathrm{0}, \mathrm{Rabb}$, and $\mathrm{Rbcd}$, then Racc and Radd.

Proof: Suppose that $a \in 0, \mathrm{Rabb}$, and Rbcd. Then, by $\mathrm{SP}_{5}$, there is some world $x$ such that Racx and Rxbd. But, by SPII, $c=x$, so Racc. By SP 7 , Rbd*c ${ }^{*}$, so by $\mathrm{SP}_{5}$ again there is a world $x$ such that $\operatorname{Rad}^{*} x$ and $\operatorname{Rxbc}^{*}$. But by SPII, $\mathrm{d}^{*}=x$, so $\operatorname{Rad}^{*} \mathrm{~d}^{*}$. Therefore, by $\mathrm{SP}_{7}$ and SP8, Radd.

This theorem tells us that around each base world there are a cluster of other worlds that are closed under the implications of the base world. Each cluster is closed under R. Our canonical model incorporates this closure in its construction. Moreover, the following theorem tells us that worlds can belong to only one cluster.

THEOREM I2 If $\mathrm{a}, \mathrm{a}^{\prime} \in 0$, Rabb, and $\mathrm{Ra}^{\prime} \mathrm{b} b$, then $\mathrm{a}=\mathrm{a}^{\prime}$.

Proof: Suppose that $a, a^{\prime} \in 0, R a b b$, and $\mathrm{Ra}^{\prime} b b$. By $\mathrm{SP}_{2}, \mathrm{Rba}^{\prime} \mathrm{b}$. So, there is a world $x$ such that Rabx and $R x a^{\prime} b$. Thus, by $S_{5}$, there is a world $y, R a a^{\prime} y$ and Rybb. By SPII, $y=a^{\prime}$. So, Raa $a^{\prime}$. By SP2, $R a^{\prime} a a^{\prime}$, and so $a \leqslant a^{\prime}$. By SPII, $a=a^{\prime}$.

In addition to the clusters, we have an elegant embedding of an $\mathrm{S}_{4}$ model in each simplified R. $\mathrm{K}^{-}$model. Suppose that $\mathcal{M}=\langle\mathrm{W}, 0, \mathrm{R}, \mathrm{S}, *, v\rangle$ is a simplified R.K $K^{-}$model. Let $\mathcal{M}^{\prime}=\left\langle 0,(T \uparrow 0),\left(*\lceil 0), v^{\prime}\right\rangle\right.$, where $v^{\prime}(p)=v(p) \cap 0$. In addition, let $\mathcal{L}^{-}$be the implication free fragment of $\mathcal{L}$. Then, we can extend $v^{\prime}$ to a satisfaction relation $\Vdash$ between worlds in 0 and formulas of $\mathcal{L}^{-}$that obeys the same clauses as those for R.K ${ }^{-}$models. First, we can show that for any $a \in 0, a \Vdash \neg A$ iff $a \nVdash A$. For, suppose that $a \in 0$. By SPI, Ra* $a^{*} a^{*}$. Then, by $\mathrm{SP}_{7}$ and SP8, Ra*aa. By SP2, Raa*a and so, by SPII, $a=a^{*}$. So, $a \Vdash \neg A$ iff a $\nVdash A$. That $T \uparrow 0$ is reflexive and transitive is easy to show.

We constrain frames to accommodate our other logics in accordance with the following correlations between schemes and semantic postulates:

\begin{tabular}{ccc}
\hline SCHEME & NAME & POStUlate \\
\hline$\square \mathrm{A} \rightarrow \diamond \mathrm{A}$ & $\mathrm{D}$ & $\exists \times T a x$ \\
$\square \mathrm{A} \rightarrow \mathrm{A}$ & $\mathrm{T}$ & $\mathrm{Saa}$ \\
$\square \mathrm{A} \rightarrow \square \square \mathrm{A}$ & 4 & $(\mathrm{Sab} \wedge \mathrm{Sbc}) \Rightarrow \mathrm{Sac}$ \\
$\mathrm{A} \rightarrow \square \diamond \mathrm{A}$ & $\mathrm{B}$ & $\mathrm{Tab} \Rightarrow \mathrm{Tba}$ \\
\hline
\end{tabular}

For soundness arguments for these schemes see [4] and [6]. 


\section{Completeness}

The completeness proof makes use of the clustering theorem $\mathrm{II}$ above and a trick from [I7] $]^{3}$ The idea is that our canonical model is made up of a set of clusters. Each cluster is centred around a normal theory. In terms of the implicational $R$ accessibility relation, the clusters are independent of one another. Worlds from one cluster are only related to worlds in other clusters by means of the modal $S$ accessibility relation. Now, once we have isolated a cluster, we make use of the trick from [ [17]. The idea is that each base world is duplicated by a non-base world. That is, each normal theory is a base world, but also, in a separate guise, acts as a non-base world. We put special restrictions on the way that base worlds behave with regard to the $R$ accessibility relation in order to verify that the canonical model is a simplified model. These restrictions would make the truth lemma (Lemma 24 below) extremely hard to prove. But having a doppelganger for each base world that behaves without the special restrictions allows us to prove it easily. For this reason we take a base world to be a pair of $\langle\Gamma, 0\rangle$ where $\Gamma$ is a normal theory. The pairing with 0 marks it out as a base world. A non-base world is a pair $\langle\Sigma, \Gamma\rangle$, where $\Sigma$ is a prime $\Gamma$-theory and $\Gamma$ is a normal theory. Thus, in the cluster around $\langle\Gamma, 0\rangle$ we also have its doppelganger $\langle\Gamma, \Gamma\rangle$. The reason that we take a non-base world to be a pair of a prime theory and a normal theory is that the normal theory indicates to which cluster the world belongs. For consider the empty theory $\emptyset$. It is a $\Gamma$-theory for every normal theory $\Gamma$. Thus, in the canonical model we have $\langle\emptyset, \Gamma\rangle$ for each such $\Gamma$.

In order to set out our canonical model, we need a few more definitions. We define a fusion operator on pairs of sets of formulas $\Sigma, \Theta$, such that $\Sigma \circ \Theta=$ $\{B: \exists A(A \rightarrow B \in \Sigma \wedge A \in \Theta)\}$. $\Sigma \circ \Theta$ is so-to-speak the modus ponens closure of $\Sigma$ and $\Theta$. Where $\Gamma$ is a normal theory and $\Sigma, \Theta, \Xi$ are sets of formulas, $R_{\Gamma} \Sigma \Theta \Xi$ if and only if for all formulas $A$ and $B$, if $\Sigma \vdash_{\Gamma} A \rightarrow B$ and $\Theta \vdash_{\Gamma} A$, then $\Xi \vdash_{\Gamma} B$.

Our canonical model is a structure $\mathcal{M}_{\mathrm{L}}=\left\langle\mathrm{W}_{\mathrm{L}}, 0_{\mathrm{L}}, \mathrm{R}_{\mathrm{L}}, \mathrm{S}_{\mathrm{L}}, *_{\mathrm{L}}, v_{\mathrm{L}}\right\rangle$ where the members of $\mathcal{M}_{\mathrm{L}}$ have the following definitions. Where $\pi_{1}$ is a projection function such that $\pi_{1}(X, Y)=X$,

- $O_{\mathrm{L}}$ is the set of pairs $\langle\Gamma, 0\rangle$ where $\Gamma$ is a normal R.K $\mathrm{K}^{-}$theory;

- $W_{\Gamma}=\left\{\langle\Sigma, \Gamma\rangle\right.$ : where $\Sigma$ is a prime $\Gamma$-theory and $\Gamma$ is a normal R.K $\mathrm{K}^{-}$ theory $\cup \cup 0_{L}$;

- $\forall a, b, c\left(W_{L} \backslash 0_{L}\right) \in R_{L} a b c \Leftrightarrow \exists \Gamma(a=\langle\Sigma, \Gamma\rangle \wedge b=\langle\Theta, \Gamma\rangle \wedge c=\langle\Xi, \Gamma\rangle \wedge$ $\Sigma \circ \Theta \subseteq \Xi)$;

- if $a \in 0_{L}: R_{L} a b c$ iff $b, c$ are $a$-theories and $b=c ; R_{L} b a c$ iff $b, c$ are $a$-theories and $b=c ; R_{L} b c a$ iff $b, c$ are $a$-theories and $b=c^{*}$;

${ }^{3}$ This is a weak completeness proof. As is well known, $\mathrm{R}$ is not strongly complete over the simplified semantics $\underline{I} 8$. 
- if $a \in 0_{L}$, then $S_{L} a b \Leftrightarrow\left(b \in 0_{L} \wedge\left(\square^{-1} a \subseteq b \subseteq \diamond^{-1} a\right)\right)$;

- if $a \notin 0_{L}, S_{L} a b \Leftrightarrow \square^{-1} a \subseteq b \subseteq \diamond^{-1} a$;

- $\forall \mathrm{a} \in\left(W_{\mathrm{L}} \backslash \mathrm{O}_{\mathrm{L}}\right)\left(\mathrm{a}=\langle\Sigma, \Gamma\rangle \Rightarrow \mathrm{a}^{*_{\mathrm{L}}}=\langle\{\mathrm{A}: \neg \mathrm{A} \notin \mathrm{a}\}, \Gamma\rangle\right) ;$

- $\forall a \in O_{L} a^{*} L=a ;$

- $v_{\mathrm{L}}(p)=\left\{a \in W_{\mathrm{L}}: p \in \pi_{1}(a)\right\}$.

Here $\square^{-1} a=\left\{A: \square A \in \pi_{1}(a)\right\}$ and $\diamond^{-1} a=\left\{A: \diamond A \in \pi_{1}(a)\right\}$.

In order to show that this construction is legitimate, we need to show the following:

LEMMA I3 If $\Gamma$ is a regular R. $K^{-}$theory and $\Sigma$ is a $\Gamma$-theory, then $\Sigma^{*}=\{\mathrm{A}: \neg A \notin \Sigma\}$ is also a $\Gamma$-theory.

Proof: Suppose that $\Gamma$ is a regular R.K ${ }^{-}$theory and $\Sigma$ is a $\Gamma$-theory. Assume that $\mathrm{B} \in \Sigma^{*}$ and $\mathrm{B} \rightarrow \mathrm{C} \in \Gamma$. Then, $\neg \mathrm{B} \notin \Sigma$ and $\neg \mathrm{C} \rightarrow \neg \mathrm{B} \in \Gamma$. So, $\neg \mathrm{C} \notin \Sigma$, hence $C \in \Sigma^{*}$. Therefore, generalizing, $\Sigma^{*}$ is a $\Gamma$-theory.

LEMMA I4 If $\Gamma$ is a normal theory, then any prime theory $\Sigma$ such that $\square^{-1} \Gamma \subseteq \Sigma \subseteq$ $\diamond^{-1} \Gamma$ is also normal.

Proof: Suppose that $\Gamma$ is a normal theory, $\Sigma$ is a prime theory, and $\square^{-1} \Gamma \subseteq$ $\Sigma \subseteq \diamond^{-1} \Gamma$. Because R.K $K^{-}$is closed under the rule of necessitation, for every theorem $A, \square A \in \Gamma$ and so $A \in \Sigma$. Thus, $\Sigma$ is regular. In addition, $\neg \diamond(A \wedge \neg A)$ is a theorem of R.K $\mathrm{K}^{-}$, so $\neg \diamond(A \wedge \neg A) \in \Gamma$ and, because $\Gamma$ is negation consistent, $\diamond(A \wedge \neg A) \notin \Gamma$. Since $\Sigma \subseteq \diamond^{-1} \Gamma$ for no formula $A,(A \wedge \neg A) \in \Sigma$. Thus, $\Sigma$ is also negation consistent. Therefore, $\Sigma$ is normal.

We now show that the canonical model is a simplified R.K ${ }^{-}$model. Sometimes, for the sake of brevity, instead of specifying, for example, that $a=\langle\Sigma, \Gamma\rangle$ and saying $A \in \Sigma$ or writing $A \in \pi_{1}(a)$ (where $\pi_{1}$ is a projection function), we will merely write ' $A \in$ a'. Similarly, we write $a \subseteq b$ instead of $\pi_{1}(a) \subseteq \pi_{1}(b)$.

LEMMA I5 If $\Sigma$ and $\Theta$ are R.K $K^{-}$theories and $\Xi$ is a prime $\Gamma$-theory such that $R_{\Gamma} \Sigma \Theta \Xi$, then there are prime $\Gamma$-theories $\Sigma^{\prime}$ and $\Phi^{\prime}$ such that $\Sigma \subseteq \Sigma^{\prime}, \Theta \subseteq \Theta^{\prime}$, and $R_{\Gamma} \Sigma^{\prime} \Theta^{\prime} \Xi$.

Proof: Suppose that $\Sigma$ and $\Theta$ are R.K ${ }^{-}$theories and $\Xi$ is a prime $\Gamma$-theory such that $R_{\Gamma} \Sigma \Theta \Xi$. Let $\Delta_{\Sigma}=\{\mathrm{B} \rightarrow \mathrm{C}: \mathrm{B} \in \Theta \wedge \mathrm{C} \notin \Xi\}$. It can be seen that $\left(\Sigma, \Delta_{\Sigma}\right)$ is $\Gamma$-independent. For suppose that there are $\mathrm{B} \rightarrow \mathrm{C}, \mathrm{B}^{\prime} \rightarrow \mathrm{C}^{\prime} \in \Delta_{\Sigma}$ such that $\Sigma \vdash_{\Gamma}(\mathrm{B} \rightarrow \mathrm{C}) \vee\left(\mathrm{B}^{\prime} \rightarrow \mathrm{C}^{\prime}\right)$. Then, by Lemma I i, $\Sigma \vdash_{\Gamma}\left(\mathrm{B} \wedge \mathrm{B}^{\prime}\right) \rightarrow\left(\mathrm{C} \vee \mathrm{C}^{\prime}\right)$. Neither $C$ nor $C^{\prime}$ is in $\Xi$ and both $B$ and $B^{\prime}$ (and hence $B \wedge B^{\prime}$ ) are in $\Theta$ by the definition of $\Delta_{\Sigma}$. Then, contrary to our assumption, it would not be that $R_{\Gamma} \Sigma \Theta \Xi$. Thus, we can extend $\Sigma$ to a prime $\Gamma$-theory $\Sigma^{\prime}$ such that $R_{\Gamma} \Sigma^{\prime} \Theta \Xi$. 
Now we extend $\Theta$ to a prime $\Gamma$-theory $\Theta^{\prime}$ such that $R_{\Gamma} \Sigma^{\prime} \Theta^{\prime} \Xi$. Let $\Delta_{\Theta}=\{A$ : $\left.\exists \mathrm{B}\left(\mathrm{A} \rightarrow \mathrm{C} \in \Sigma^{\prime} \wedge \mathrm{C} \notin \Xi\right)\right\}$. We can see that $\left(\Theta, \Delta_{\Theta}\right)$ is $\Gamma$-independent. For suppose that $\Theta \vdash_{\Gamma}\left(A \vee A^{\prime}\right)$ for some $A, A^{\prime} \in \Delta_{\Theta}$. Then there are $C, C^{\prime} \notin \Xi$ such that $A \rightarrow C \in \Sigma^{\prime}$ and $A^{\prime} \rightarrow C^{\prime} \in \Sigma^{\prime}$. Then, by axioms 5 and $7,\left(\left(A \vee A^{\prime}\right) \rightarrow\right.$ $\left.\left(C \vee C^{\prime}\right)\right) \in \Sigma^{\prime}$. Since $\Xi$ is prime, $C \vee C^{\prime} \notin \Xi$. Thus, it cannot be the case that $R_{\Gamma} \Sigma^{\prime} \Theta \Xi$. So, $\left(\Theta, \Delta_{\Theta}\right)$ is $\Gamma$-independent. Now we extend $\Theta$ to a prime theory $\Theta^{\prime}$ such that $R_{\Gamma} \Sigma^{\prime} \Theta^{\prime} \Xi$ ending the proof of the lemma.

We begin by showing that SPII holds, because proving this makes it redundant to show that $\mathrm{SP}_{4}, \mathrm{SP}_{5}, \mathrm{SP}_{6}$, and $\mathrm{SP}_{9}$ hold, since they follow automatically from SPII.

LEMMA I6 (SPI3) For all worlds $\mathrm{a}$ and $\mathrm{b}$ in $\mathrm{W}_{\mathrm{L}}, \mathrm{a} \leqslant_{\mathrm{L}} \mathrm{b}$ iff $\mathrm{a}=\mathrm{b}$.

Proof: $\Leftarrow$ trivial. $\Rightarrow$ Suppose that $a \leqslant L$ b, i.e. there is a world $x$ in $0_{L}$ such that $R_{L} x a b$. Then, by the construction of the canonical model, $a=b$.

We also prove $\mathrm{SP}_{7}$ and $\mathrm{SP} 8$ out of order because we assume them in later lemmas.

LEMMA I7 $\left(\mathrm{SP}_{7}\right) \mathrm{R}_{\mathrm{L}} a b c \Rightarrow \mathrm{R}_{\mathrm{L}} a c^{* \mathrm{~L}} b^{* \mathrm{~L}}$.

Proof: Suppose that $R_{\mathrm{L}} a b c$.

Case I. $a, b, c \notin O_{L}$ (so $b^{* L}, c^{* L} \notin 0$ ). Suppose that $A \rightarrow B \in a$ and $A \in c^{* L}$. Then, $\neg A \notin \mathrm{c}$. But, by axioms $\mathrm{I}_{2}$ and $\mathrm{I} 3, \neg \mathrm{B} \rightarrow \neg A \in \mathrm{a}$. So $\neg \mathrm{B} \notin \mathrm{b}$, hence $\mathrm{B} \in \mathrm{b}^{* \mathrm{~L}}$. Generalizing, $\mathrm{R}_{\mathrm{L}} a c^{* \mathrm{~L}} \mathrm{~b}^{* \mathrm{~L}}$.

Case 2. $a \in 0_{L}$. Then, by the construction of the canonical model, $b=c$ and $R_{\mathrm{L}} a b^{*} \mathrm{~L}^{* \mathrm{~L}}$.

Case 3. $b \in 0_{L}$. Then, $a=c$, so $R_{L} a b a$. Moreover, $b=b^{*}$ and by the definition of $R_{L}, R_{L} a a^{*} b$.

Case 4. $c \in 0_{L}$. Then, $a=b^{*}$ and $c=c^{*}$. By the definition of $R_{L}, R_{L} a c a$, i.e. $R_{\mathrm{L}} a c^{*}{ }^{*} b^{*} \mathrm{~L}$.

LEMMA I8 (SP8) For all worlds a in $\mathrm{W}_{\mathrm{L}}, \mathrm{a}^{\mathrm{L}_{\mathrm{L}}{ }^{*} \mathrm{~L}}=\mathrm{a}$.

Proof: Case I. $a \in 0_{L}$. Then $a^{*}=a$, by definition. Case 2. $a \notin 0_{L}$. Suppose that $A \in \mathrm{a}^{{ }^{\mathrm{L}} *_{\mathrm{L}}}$. Then, $\neg A \notin \mathrm{a}^{* \mathrm{~L}}$, so $\neg \neg A \in \mathrm{a}$. By axiom $\mathbf{2}, A \in$ a. Suppose now that $A \in \mathrm{a}$. Then, by axiom I2 $\neg \neg A \in \mathrm{a}$. So, $\neg A \notin \mathrm{a}^{* \mathrm{~L}}$ and $\neg \neg A \in \mathrm{a}^{*{ }^{*} \mathrm{~L}}$. Hence by axiom $12 A \in a^{*{ }^{*} L}$.

LEMMA I9 (SPI) For all worlds a in $\mathrm{W}_{\mathrm{L}}, \mathrm{R}_{\mathrm{L}}$ aaa.

Proof: By Lemma国ii, $\vdash((A \rightarrow B) \wedge A) \rightarrow B$. So, suppose that $A \rightarrow B \in a$ and $A \in a$. Then, $B \in a$. Generalizing, $a \circ a \subseteq a$, hence $R_{L}$ aaa.

LeMma $20\left(\mathrm{SP}_{2}\right)$ For all worlds $\mathrm{a}, \mathrm{b}$ and $\mathrm{c}$ in $\mathrm{W}_{\mathrm{L}}$, if $\mathrm{R}_{\mathrm{L}} \mathrm{abc}$, then $\mathrm{R}_{\mathrm{L}}$ bac. 
Proof: Suppose that $R_{\mathrm{L}} a b c$. By the construction of the canonical model, there is some normal theory $\Gamma$ such that $a=\langle\Sigma, \Gamma\rangle, b=\langle\Theta, \Gamma\rangle$, and $c=\langle\Xi$, $\Gamma\rangle$ and $\Sigma \circ \Theta \subseteq \Xi$. Now suppose that $A \rightarrow B \in \Theta$ and $A \in \Sigma$. By axiom 3, $(A \rightarrow B) \rightarrow$ $B \in \Sigma$. So, since $\Sigma \circ \Theta \subseteq \Xi, B \in \Xi$. Generalizing, $\Theta \circ \Sigma \subseteq \Xi$. So, by the definition of $R_{L}, R_{L}$ bac.

LEMMA 2I $\left(\mathrm{SP}_{3}\right)$ If there is a world $x$ such that $\mathrm{R}_{\mathrm{L}} a b x$ and $\mathrm{R}_{\mathrm{L}} \mathrm{xcd}$, then there is a world $y$ such that $\mathrm{R}_{\mathrm{L}}$ acy and $\mathrm{R}_{\mathrm{L}} \mathrm{ybd}$.

Proof: Suppose that there is a world $x$ such that $R_{L} a b x$ and $R_{L} x c d$.

Case o. None of $a, b, x, c, d$ are in $O_{L}$. By the construction of the canonical model, there is a normal theory $\Gamma$ such that $a, b, x, c, d$ are all based on $\Gamma$-theories and $\mathrm{a} \circ \mathrm{b} \subseteq \mathrm{x}$ and $\mathrm{x} \circ \mathrm{c} \subseteq \mathrm{d}$. We show that $(\mathrm{a} \circ \mathrm{c}) \circ \mathrm{b} \subseteq \mathrm{d}$. Suppose that $C \in(a \circ c) \circ b$. Then there is $a B \in b$ such that $B \rightarrow C \in a \circ c$ and so there is an $A \in c$ such that $A \rightarrow(B \rightarrow C) \in a$. By axiom $4, B \rightarrow(A \rightarrow C) \in$ a. Then, since $\mathrm{a} \circ \mathrm{b} \subseteq \mathrm{x}, \mathrm{A} \rightarrow \mathrm{C} \in \mathrm{x}$. Moreover, since $\mathrm{x} \circ \mathrm{c} \subseteq \mathrm{d}, \mathrm{C} \in \mathrm{d}$. Generalizing $(\mathrm{a} \circ \mathrm{c}) \circ \mathrm{b} \subseteq \mathrm{d}$. Now, by Lemma I5, we can extend $\mathrm{a} \circ \mathrm{c}$ to a prime $\Gamma$-theory such $y$ that $R_{L} y b d$ and $R_{L} a c y$.

Case I. $a \in O_{L}$. Then, $x=b$. So we have $R_{L} a b b$ and $R_{L} b c d$. But, by the construction of the canonical model, $R_{\mathrm{L}}$ acc. Moreover, by Lemma 20 , $R_{\mathrm{L}} c b d$. Hence there is a world $y$ such that $R_{L}$ acy and $R_{L} y b d$.

Case 2. $b \in 0_{L}$. Then $a=x$ and we have $R_{L} a b a$ and $R_{L} a c d$. By the construction of the canonical model, we have $R_{L}$ bdd and, so by Lemma 20 , $R_{L} d b d$. But we already have $R_{L} a c d$, so there is a world $y$, namely $d$, such that $R_{L}$ acy and $R_{L} y b d$.

Case 3. $x \in 0_{L}$. Then $b=a^{* L}$ and $d=c^{*}$. That is, $R_{L} a a^{*}{ }^{2} x$ and $R_{L} x c c^{* L}$. Let $x=\langle\Gamma, 0\rangle$ and let $x^{\prime}=\langle\Gamma, \Gamma\rangle$. By the construction of the canonical model, $R_{L} a a^{*} x^{\prime}$ and $R_{L} x^{\prime} c c^{*}{ }^{*}$. The rest proceeds as in the other cases.

Case 4. $c \in O_{L}$. Then we have $x=d$ and $R_{L} a b d$. By the construction of the canonical model, we know that $R_{L} a c a$. Thus, there is a world $y$, namely a, such that $R_{L}$ acy and $R_{L} y b d$.

Case 5. $d \in 0_{L}$. Then we know that $x=c^{*}$ so we have $R_{L} a b c^{*}$ and $R_{L} c^{*} \mathrm{~L} c d$. So, by Lemma 20 , we know that $R_{L} a c b^{*}$ and, by the construction the canonical model, $R_{L} b^{*} \mathrm{~b} d$. So, we have a world $y$, namely $b^{*_{L}}$, such that $R_{L} a c y$ and $R_{L} y b d$.

LEMma 22 For all $a \in W_{\mathrm{L}}$, and all formulas $\mathrm{A}, \mathrm{B}, \mathrm{A} \rightarrow \mathrm{B} \in \mathrm{a}$ iff for all $\mathrm{b}, \mathrm{c} \in \mathrm{W}_{\mathrm{L}}$, if $\mathrm{R}_{\mathrm{L}} \mathrm{abc}$ and $\mathrm{A} \in \mathrm{b}$, then $\mathrm{B} \in \mathrm{c}$.

Proof: $\Rightarrow$ by definition of $R_{\mathrm{L}}$.

$\Leftarrow$ Suppose that $A \rightarrow B \notin$ a. Case I. $a \in 0_{L}$, that is, $a$ is $\langle\Gamma, 0\rangle$, where $\Gamma$ is a normal R.K $K^{-}$theory. Then, $(\{A\},\{B\})$ is $\Gamma$-independent. So, by Lemma 2 , there is a prime $\Gamma$-theory $b$ that contains $A$ and does not contain $B$. Since $b$ is a prime $\Gamma$-theory, $R_{L} a b b$. 
Case 2. $a \notin 0_{L}$. Let $a=\langle\Sigma, \Gamma\rangle$. Then $(a \circ\{A\},\{B\})$ is $\Gamma$-independent, for if $a \circ\{A\} \vdash_{\Gamma} B$, then there would be a formula $C$ such that $A \rightarrow C \in a$ and $\mathrm{C} \rightarrow \mathrm{B} \in \Gamma$. But since $\mathrm{a}$ is a $\Gamma$-theory, $\mathrm{A} \rightarrow \mathrm{B}$ would be in a, contrary to the hypothesis. By Lemma 2 we can extend $a \circ\{A\}$ to $\Theta$, a prime $\Gamma$-theory that does not include B. Let us call $\langle\Theta, \Gamma\rangle$, c. So now we have $R_{\Gamma} \Sigma\{A\} \Theta$ where $\Sigma$ and $\Theta$ are prime $\Gamma$-theories. So, by Lemma 15 , there is a prime $\Gamma$-theory $\Xi$ extending $\{A\}$ such that $R_{\Gamma} \Sigma \Xi \Theta$. Let us call $\langle\Xi, \Gamma\rangle$, b. So we have $R_{L} a b c$.

LEMMA 23 For all $a \in W_{L}$, and all formulas $A, \square A \in a$ iff for all $b \in W_{L}$, if $S_{L} a b$, $A \in b$.

Proof: $\Rightarrow$ By definition of $S_{L}$.

$\Leftarrow$ Suppose that $\square A \notin a$. Let us set $\Delta=\{A\} \cup\{B: \diamond B \notin a\}$. $\left(\square^{-1} a, \Delta\right)$ is independent. For suppose that $\square^{-1} a \vdash_{R . K^{-}} \Delta$. Then, we would have $C_{1}, \ldots, C_{n} \in \square^{-1} a$ and $B_{1}, \ldots, B_{m} \in \Delta$ such that $\vdash\left(C_{1} \wedge \ldots \wedge C_{n}\right) \rightarrow$ $\left(A \vee B_{1} \vee \cdots \vee B_{m}\right)$. By necessitation and the distribution of necessity over implication, we then get $\vdash \square\left(C_{1} \wedge \ldots \wedge C_{n}\right) \rightarrow \square\left(A \vee B_{1} \vee \cdots \vee B_{m}\right)$. But $\vdash \square\left(A \vee B_{1} \vee \cdots \vee B_{m}\right) \rightarrow\left(\square A \vee \diamond B_{1} \vee \cdots \vee \diamond B_{m}\right)$, so we have $\vdash \square\left(C_{1} \wedge\right.$ $\left.\ldots \wedge C_{n}\right) \rightarrow\left(\square A \vee \diamond B_{1} \vee \cdots \vee \diamond B_{m}\right)$. But $\square\left(C_{1} \wedge \cdots \wedge C_{n}\right) \in a$, by hypothesis and axiom 18 , so $\left(\square A \vee \diamond B_{1} \vee \cdots \vee \diamond B_{m}\right) \in a$. Moreover, $a$ is prime, so we have $\square A \in a$ or $\diamond B_{1} \in a$, or...or $\diamond B_{m} \in a$. But none of these formulas is in $a$. So, by reductio, $\left(\square^{-1} a, \Delta\right)$ is independent. Then by Lemma 2 , we can extend $\square^{-1}$ a to a prime R.K $\mathrm{K}^{-}$theory $\Sigma$ such that $\Sigma \cap \Delta=\emptyset$.

Now we construct a normal theory $\Gamma$ such that $\Sigma$ is a $\Gamma$-theory. Let $\Gamma^{\prime}$ be the set of R.K $\mathrm{K}^{-}$Theorems. Then, we know that $\mathrm{R}_{\Gamma^{\prime}} \Gamma^{\prime} \Sigma \Sigma$. By Lemma I5, there is a prime extension $\Gamma^{\prime \prime}$ of $\Gamma$ such that $R_{\Gamma^{\prime}} \Gamma^{\prime \prime} \Sigma \Sigma$. In [8], moreover, it is shown that each prime regular R.K $\mathrm{K}^{-}$theory has a normal theory as a subset. Let us call a normal theory contained in $\Gamma^{\prime \prime}, \Gamma$. Then $R_{\Gamma^{\prime}} \Gamma \Sigma \Sigma$. Now we set $\mathrm{b}=\langle\Sigma, \Gamma\rangle$. Thus, there is $a b$ in $W_{L}$ such that $S_{L} a b$ and $A \notin b$.

Now we can prove the usual truth lemma.

LEMMA 24 (Truth) For all worlds $\mathrm{a} \in \mathrm{W}_{\mathrm{L}}$ and all formula $\mathrm{A}, \mathrm{a} \models_{\mathrm{L}} \mathrm{A}$ iff $\mathrm{A} \in \mathrm{a}$.

Proof: By induction on the complexity of $A$.

Case I. $A=p$. By definition of $\models_{\mathrm{L}}$.

Case 2. $A=B \wedge C$. Easy.

Case 3. $A=B \vee C$. By the inductive hypothesis and the primeness of worlds.

Case 4. $A=B \rightarrow C$. By the inductive hypothesis and Lemma 22 .

Case 5. $A=\square B$. By the inductive hypothesis and Lemma 23 .

THEOREM 25 For all formula A, $\vdash_{\text {R.K- }}$ A iff for all R.K $K^{-}$models $\mathcal{M}, \mathcal{M} \models A$.

Proof: $\Rightarrow$ by soundness. $\Leftarrow$ Suppose that for all R.K $\mathrm{K}^{-}$models $\mathcal{M}, \mathcal{M} \models A$. The canonical model is an R.K $\mathrm{K}^{-}$model. Thus, $\mathcal{M}_{\mathrm{L}} \models A$. Then, by Lemma 24, 
$A \in \Gamma$ for all normal theories $\Gamma$. By Lemma 9 , if $A$ is in every normal theory, then $\vdash_{\text {R.K }}-A$.

\section{Boolean Negation}

The point of the completeness proof is to aid in proving that the addition of boolean negation to R.K $\mathrm{K}^{-}$yields a conservative extension. First we add boolean negation, $\sim$, to our language, creating $\mathcal{L}^{\sim}$. We construct a boolean extension, CR.K ${ }^{-}$, of R.K ${ }^{-}$by adding the following axiom schemes 4

$$
\begin{aligned}
& \text { C.I } A \rightarrow(B \rightarrow(C \vee \sim C)) \\
& \text { C. } 2 A \rightarrow(\sim(B \rightarrow C) \vee(\sim B \vee C)) \\
& \text { C. } 3(A \wedge \sim A) \rightarrow B \\
& \text { C. } 4 \diamond(A \wedge \sim A) \rightarrow B \\
& \text { C. } 5 \rightarrow \square(B \vee \sim B)
\end{aligned}
$$

A CR. $\mathrm{K}^{-}$model is just a simplified R.K ${ }^{-}$model. The difference between the semantics for the two logics (and two languages) is that the satisfaction relation is slightly different. To mark out the difference with R.K $\mathrm{K}^{-}$, we use $\Vdash$ for a CR.K ${ }^{-}$satisfaction relation. The definition of $\Vdash$ agrees with its corresponding $\models$ on all clauses except, of course, that the definition for $\Vdash$ has an extra clause for boolean negation, that is,

$$
a \Vdash \sim A \text { iff } a \nVdash A \text {. }
$$

The notion of validity remains as it is for R.K ${ }^{-}$.

We first prove soundness.

TH EOREM 26 (Soundness) Every instance of axioms C.I-C.5 are valid on all simplified models for CR.K. ${ }^{-}$.

Proof: We note first that $A \vee \sim A$ is true at every world and that $A \wedge \sim A$ fails at every world.

C.I. Suppose that $a \Vdash A$ and that Rabc and $b \Vdash B$. As we have noted, $c \Vdash C \vee \sim C$. Hence, by the truth condition for implication, $a \Vdash B \rightarrow$ $(C \vee \sim C)$ and, by Lemma IO, $A \rightarrow(B \rightarrow(C \vee \sim C))$ is valid.

C.2. Suppose that $a \Vdash A$. Also assume that $a \nVdash \sim(B \rightarrow C)$, i.e., $a \Vdash(B \rightarrow C)$. By SPI, Raaa, so either a $\nVdash B$ or $a \Vdash C$, that is a $\Vdash \sim B \vee C$. Hence a $\Vdash \sim($ $\mathrm{B} \rightarrow \mathrm{C}) \vee(\sim \mathrm{B} \vee \mathrm{C})$. Thus, by Lemma $\mathrm{IO} A \rightarrow(\sim(\mathrm{B} \rightarrow \mathrm{C}) \vee(\sim \mathrm{B} \vee \mathrm{C}))$ is valid.

\footnotetext{
${ }^{4}$ The first three of these are taken from [II].
} 
C.3. $(A \wedge \sim A)$ is never true at any world. So, vacuously, by Lemma IO $(A \wedge \sim A) \rightarrow B$ is valid.

C.4. Since $(A \wedge \sim A)$ is never true, nor is $\diamond(A \wedge \sim A)$, so $\diamond(A \wedge \sim A) \rightarrow B$ is valid.

C.5. $\square(B \vee \sim B)$ is true at every world, hence $A \rightarrow \square(B \vee \sim B)$ is valid.

Now we prove conservative extension.

LEMMA 27 Let $\mathrm{F}$ be a simplified R.K $K^{-}$frame and $\models$ an R.K $K^{-}$valuation and $\Vdash a$ $C R . K^{-}$valuation on $F$ such that for all propositional variables $p$ and all worlds a in $F, \mathrm{a} \Vdash \mathrm{p}$ iff $\mathrm{a} \models \mathrm{p}$. Then for all formulas $\mathrm{A}$ not containing boolean negation, $\mathrm{a} \Vdash \mathrm{A}$ iff $a \models A$, for all worlds $a$.

Proof: By a simple induction on the complexity of $A$.

THEOREM 28 Let A be a formula not containing boolean negation. Then, $\vdash_{\mathrm{CR} . \mathrm{K}^{-}} \mathrm{A}$ iff $\vdash_{\text {R.K }}-A$.

Proof: $\Leftarrow$ by the fact that all the axioms of $\mathrm{R}^{-\mathrm{K}^{-}}$are axioms of CR.K ${ }^{-}$and the rules of R.K ${ }^{-}$are all rules of CR.K ${ }^{-}$.

$\Rightarrow$ Suppose that $\nvdash_{\text {R. } K^{-}}$A. Consider the frame $\mathcal{F}_{\mathrm{L}}$ of the canonical model. Let us construct a canonical CR. $\mathrm{K}^{-}$valuation, $\Vdash_{\mathrm{L}}$. We set $a \Vdash_{\mathrm{L}} p$ iff $a \models_{\mathrm{L}} \mathrm{p}$, for all worlds a and all propositional variables $p$. Then, by Lemma 27, we have $a \Vdash_{L} B$ iff $a \models_{L} B$ for all formulas B not containing boolean negation. Consider one such formula $A$ that is not a theorem of R.K ${ }^{-}$. By Lemma 9, there is a normal theory $\Gamma$ that does not contain $A$. By the construction of the canonical model, $\langle\Gamma, \Gamma\rangle \in 0_{\mathrm{L}}$. By Lemma 24, $\langle\Gamma, \Gamma\rangle \nvdash_{\mathrm{L}} A$. So, $\langle\Gamma, \Gamma\rangle \nVdash_{\mathrm{L}} A$. Thus, $A$ is not CR. $\mathrm{K}^{-}$-valid. So, by theorem $26, \nvdash_{\mathrm{CR} . \mathrm{K}^{-}}$A.

THEOREM 29 The boolean extension of any of the extensions of R. $K^{-}$by any collection of the schemes $D, T, 4$, and $B$ is conservative over the corresponding non-boolean logic.

Proof: Follows directly from the completeness theorem for the logic in question and Lemma 27

Thus we have proven that CR.K $\mathrm{K}^{-}$is a conservative extension of R.K ${ }^{-}$. Extending this proof to the other logics is straightforward.

\section{REFERENCES}

[i] A.r. anderson, N.D. Belnap, and J.m. dunn, The Semantics of Entailment, volume II, Princeton: Princeton University Press, 1992

[2] R.T. BRADY (ED.), Relevant Logic and its Rivals, volume II, Aldershot: Ashgate, 2003 
[3] J.M. DUnN "A 'Gentzen' System for Positive Relevant Implication” The fournal of Symbolic Logic 38 pp. 356-357

[4] A. Fuhrmann "Models for Relevant Modal Logics" Studia Logica 49 (1990)

[5] L. GOble "Deontic Logic with Relevance" in P. McNamara and H. Prakken (eds.), Norms, Logics, and Information Systems, Amsterdam: IOs Press, I999, pp. 33I-345

[6] E.D. Mares "Classically Complete Modal Relevant Logics" Mathematical Logic Quarterly 39 (1993) pp. I65-177

[7] E.D. MAREs "Mostly Meyer Modal Models" Logique et analyse I46 (1994) pp. II9-I28

[8] E.D. mares and R.K. Meyer "The Admissibility of $\gamma$ in R4" Notre Dame Fournal of Formal Logic 33 (1992) pp. 197-206

[9] E.D. MAREs "CE is not a Conservative Extension of E" fournal of Philosophical Logic 29 (2000) pp. 263-275

[IO] E.D. MARES and R.K. MEYER "The Semantics of R4" Fournal of Philosophical Logic 22 (1993) pp. 95-IIO

[ir] R.K. Meyer and S. giambrone "Completeness and Conservative Extension Results for some Boolean Relevant Logics” Studia Logica 48 (1989) pp. I-I4

[I2] R.K. MEYER and E.D. MARES "The Semantics of Entailment O" in K. Došen and P. Schroder-Heister (eds.), Substructural Logics, Oxford: Oxford University Press, 1993, pp. 239-258

[13] R.K. meyer and R. Routley "Classical Relevant Logics i" Studia Logica 32 (I973) pp. 5I-68

[I4] R.K. MEYer and R. ROUtLey "Classical Relevant Logics I I" Studia Logica 33 (1974) pp. I83-94

[I5] G. MINTs "Systemy Lyuisa i sistema T" (in Russian) Supplement to the Russian translation of в. FEYs, Modal Logic, Moscow: Nauka, 1974, pp. 422-509. English translation in G. MINTs, Selected Papers in Proof Theory, Amsterdam: North Holland, 1992

[I6] G. PRIEST and R. SYlvan "Simplified Semantics for Basic Relevant Logics" Fournal of Philosophical Logic 2I (1992) pp. 217-232

[I7] G. RESTALL "Simplified Semantics for Relevant Logics (and some of their Rivals)" Gournal of Philosophical Logic 22 (I993) pp. 48I-5II 
[18] G. Restall and T. ROy "On Permutation in Simplified Semantics," Fournal of Philosophical Logic, 38:3 (2009) pp. 333-34I

[ig] R. ROUTLEY and R.K. MEYER "The Semantics of Entailment i I" fournal of Philosophical Logic I (1972) pp. 53-73

[20] к. тANaka "To Be Something and Something Else: Dialethic Tense Logic" Logique et analyse I6I-I62-I63 (1998) pp. I89-202 
The Australasian fournal of Logic (ISSN I448-5052) disseminates articles that significantly advance the study of logic, in its mathematical, philosophical or computational guises. The scope of the journal includes all areas of logic, both pure and applied to topics in philosophy, mathematics, computation, linguistics and the other sciences.

Articles appearing in the journal have been carefully and critically refereed under the responsibility of members of the Editorial Board. Only papers judged to be both significant and excellent are accepted for publication.

The journal is freely available at the journal website at

$$
\text { http://www.philosophy.unimelb.edu.au/ajl/ }
$$

All issues of the journal are archived electronically at the journal website.

SuBSCRIPTIONS Individuals may subscribe to the journal by sending an email, including a full name, an institutional affiliation and an email address to the managing editor at ajl-editors@unimelb.edu.au Subscribers will receive email abstracts of accepted papers to an address of their choice. For institutional subscription, please email the managing editor at ajl-editors@unimelb.edu.au

Complete published papers may be downloaded at the journal's website at http: //www.philosophy.unimelb.edu.au/ajl/ The journal currently publishes in pdf format.

Submission The journal accepts submissions of papers electronically. To submit an article for publication, send the $\mathrm{LT}_{\mathrm{E}} \mathrm{X}$ source of a submission to a member of the editorial board. For a current list of the editorial board, consult the website.

The copyright of each article remains with the author or authors of that article. 